\title{
Regulation of the key antioxidant enzymes by developmental processes and environmental stresses in the dark
}

\author{
P. POÓR ${ }^{1 *}$, A. ÖRDÖG ${ }^{1}$, Z. CZÉKUS ${ }^{1,2}$, P. BORBÉLY ${ }^{1,2}$, Z. TAKÁCS ${ }^{1}$, J. KOVÁCS $^{1}$, and I. TARI ${ }^{1}$ \\ Department of Plant Biology, University of Szeged, H-6726 Szeged, Hungary ${ }^{1}$ \\ Doctoral School in Biology, Faculty of Science and Informatics, University of Szeged, H-6726 Szeged, Hungary ${ }^{2}$
}

\begin{abstract}
The presence of solar radiation is one of the most important environmental factors, which is required for the optimal growth and development of plants. The absence of it (e.g. in the night period or artificially prolonged darkness) can alter the light-dependent signalling and regulation pathways and may induce new defence responses. Antioxidant enzymes as components of the plant defence system play a crucial role in the detoxification of reactive oxygen species (ROS) induced by several stressors; however, their regulation can be different in the light or in the dark. In this review we summarize the current knowledge about the physiological and molecular aspects of dark-modulated key antioxidant enzymes (superoxide dismutase, catalase, and ascorbate peroxidase) in different plant species and discuss their roles in different developmental processes (seedling growth and development or senescence) and in responses to environmental stresses (cold, chilling, heat, and biotic stress). Moreover, the hormonal regulation of respective gene transcription and the changes in activity of various isoenzymes at subcellular level are also summarized. Based on this knowledge, modification of these antioxidant enzymes may be used to increase the yield and stress tolerance of cultivated plants in the changing environment.
\end{abstract}

Additional key words: ascorbate peroxidase, catalase, reactive oxygen species, superoxide dismutase.

\section{Introduction}

Plants are able to sense and respond to the changing environment by modifying their growth and development. The presence or absence of solar or artificial radiation is one of the most important environmental factors. Optimal irradiance is required for growth and development, and may also control the plant defence mechanisms. Moreover, excess of radiation leads to oxidative stress, which may contribute to the initiation of cell death in leaf tissues, whereas the absence of radiation may modulate plant responses or induce leaf senescence (Karpinski et al. 2003, Kangasjärvi et al. 2012, LuschinEbengreuth and Zechmann 2016). Plants are able to sense the quantity, quality, and direction of solar radiation as well as changes in the photoperiod (Chen et al. 2004,

Kazan and Manners 2011). Hence, the absence of radiation (e.g., in the night or under prolonged darkness) can alter the light-dependent activation of plant defence responses and induce new signalling and regulation pathways modulated by phytohormones (Ballaré 2014). Thus, the regulation of plant responses to diverse environmental stimuli seems to be different under light and dark conditions.

The aim of this review was to summarize the current knowledge on the regulation of the key antioxidant enzymes in plant developmental processes and stress responses under dark conditions, because these enzymes may play a crucial role in the regulation of detoxification and homeostasis of reactive oxygen species (ROS).

Submitted 4 July 2017, last revision 13 October 2017, accepted 16 November 2017.

Abbreviations: ABA - abscisic acid; APX - ascorbate peroxidase, AsA - ascorbate; CAT - catalase, CKs - cytokinins; DHA - dehydroascorbate; ET - ethylene; ETC - electron transport chains; GA - gibberellic acid; GR - glutathione reductase; GSH - glutathione; GSSG - glutathione disulfide; MDHA - monodehydroascorbate; NAA - naphthalene acetic acid; NO - nitric oxide; ROS - reactive oxygen species; SOD - superoxide dismutase.

Acknowledgments: We apologize to those colleagues whose work was not reviewed here. This work was supported by grants from the Hungarian Scientific Research Fund (OTKA PD112855) and by the UNKP-17-4 New National Excellence Program of Ministry of Human Capacities and by the EU-funded Hungarian Grant EFOP-3.6.1-16-2016-00008.

* Corresponding author; e-mail: poorpeti@bio.u-szeged.hu 


\section{Basic properties of the key antioxidant enzymes}

A wide range of biotic or abiotic stressors can cause deregulation, overflow or even disruption of the electron transport chains (ETC) in mitochondria and chloroplasts, and thus may induce rapid production and accumulation of reactive oxygen species (ROS) in plant tissues. Under these conditions, singlet oxygen $\left({ }^{1} \mathrm{O}_{2}\right)$, hydroxyl radical $(\mathrm{OH})$, superoxide radical $\left({ }^{\circ} \mathrm{O}_{2}^{-}\right)$, and hydrogen peroxide $\left(\mathrm{H}_{2} \mathrm{O}_{2}\right)$ can be formed, which are strongly oxidizing compounds and therefore they can be harmful for cell integrity (Foyer and Noctor 2009). Among them, $\mathrm{H}_{2} \mathrm{O}_{2}$ is the most stable ROS (with a half-life of $1 \mathrm{~ms}$ ); it is diffusible and can act as a toxic compound (inducing oxidative stress) or as a signalling molecule (mediating tolerance by inducing antioxidant enzymes) (Vranová et al. 2002, Kocsy et al. 2013, Van Aken and Van Breusegem 2015).

To minimize the harmful effects of ROS, plants have evolved sophisticated defence mechanisms, which can control ROS accumulation, for example, various antioxidant systems such as the Foyer-Halliwell-Asada cycle (Foyer and Noctor 2009). There are three key antioxidant enzymes, which play a crucial role in ROS detoxification. The first is superoxide dismutase (SOD, EC 1.15.1.1), which converts ${ }^{\circ} \mathrm{O}_{2}{ }^{-}$to $\mathrm{O}_{2}$ and $\mathrm{H}_{2} \mathrm{O}_{2}$. The $\mathrm{O}_{2}{ }^{-}$can be produced at all locations where an electron transport chain operates (e.g., in mitochondria and chloroplasts) and in some other cell compartments including microsomes, glyoxysomes, peroxisomes, or even in the apoplast or cytosol. For this reason, SODs are present in all these compartments. Based on their metal co-factors, SODs are classified into three groups in plants: FeSODs, MnSODs, Cu/ZnSODs. FeSODs can be further divided to two groups: the homodimers formed by two identical $20 \mathrm{kDa}$ subunit proteins, with iron in the active centre and tetramers with four equal subunits with a molecular mass of 80 - $90 \mathrm{kDa}$, also containing iron in the active centre. FeSODs are inactivated by $\mathrm{H}_{2} \mathrm{O}_{2}$ and they are resistant to potassium cyanide. MnSODs are either homodimers or homotetramers with one $\mathrm{Mn}$ (III) atom per subunit. Moreover, MnSODs are neither inhibited by $\mathrm{KCN}$, nor inactivated by $\mathrm{H}_{2} \mathrm{O}_{2}$. $\mathrm{Cu} / \mathrm{ZnSODs}$ can also be divided to two different groups: the first is homodimeric, whereas the second is homotetrameric. FeSODs are located in the chloroplasts, MnSODs are present in the mitochondria and peroxisomes, whereas $\mathrm{Cu} / \mathrm{ZnSODs}$ can be found in the chloroplasts, cytosol, or apoplast (Alscher et al. 2002).

Hydrogen peroxide can be mostly degraded by catalase (CAT) and ascorbate peroxidase (APX) but also by several other enzymes (e.g., other peroxidases) to $\mathrm{H}_{2} \mathrm{O}$ (Foyer and Noctor 2009). CATs (EC 1.11.1.6) are predominantly heme-containing tetrameric proteins of about $220 \mathrm{kDa}$ molecular mass, but there are also some exceptions: non-heme CATs, dimeric CATs, or CATs with a much higher molecular mass (Foyer and Noctor 2000). In Arabidopsis thaliana there are three CAT genes, two of which are located on chromosome 1 (CAT1,
CAT3) and one is located on chromosome 4 (CAT2). However, based on expression and functional analyses, CATs can be divided into three classes (Mhamdi et al. 2010). It was found that transcriptions of class I and class II display opposing day-night rhythms, and they are under circadian regulation in many plants (Mhamdi et al. 2010). Basically, the highest CAT activity is found in the peroxisomes. Additionally, mitochondrial and chloroplastic forms of CATs exist in maize and spinach (Foyer and Noctor 2000).

The third key ROS detoxifying enzyme is ascorbate peroxidase (APX, EC 1.11.1.11). APX belongs to the class I heme peroxidase superfamily, which plays a role in $\mathrm{H}_{2} \mathrm{O}_{2}$ scavenging (Asada 1997, Wada et al. 2003, Caverzan et al. 2012). APX has a higher affinity to $\mathrm{H}_{2} \mathrm{O}_{2}$ and is able to detoxify low concentrations of $\mathrm{H}_{2} \mathrm{O}_{2}$, whereas CAT has a high reaction rate but a low affinity to $\mathrm{H}_{2} \mathrm{O}_{2}$. Eight types of APX have been described in Arabidopsis: three cytosolic (APX1, APX2, APX6), two chloroplastic (stromal sAPX, thylakoid tAPX) and three microsomal (APX3, APX4, APX5) isoforms. Microsomal isoenzymes are involved in $\mathrm{H}_{2} \mathrm{O}_{2}$ detoxification produced by $\beta$-oxidation of fatty acids especially during seed germination or by photorespiration. Chloroplastic APXs can protect the photosynthetic apparatus against oxidative stress, whereas the cytosolic APX isoenzymes have general stress-protective functions (Panchuk et al. 2005). Therefore, the individual APX isoenzymes seem to play specific physiological role at particular subcellular level and their activities can be regulated by irradiance.

The nonenzymatic elements of the Foyer-HalliwellAsada cycle are ascorbate (AsA)/dehydroascorbate (DHA) and glutathione (GSH)/glutathione disulfide (GSSG) cycles. AsA is oxidized by APX to monodehydroascorbate (MDHA) and DHA. It has to be also mentioned that both AsA and GSH can be directly oxidized by ROS, however, these reactions show slow kinetics. In the regeneration part of the pathway, MDHA reductase (MDHAR, EC 1.6.5.4), DHA reductase (DHAR, EC 1.8.5.1) and glutathione reductase (GR, EC 1.6.4.2) recycle the oxidized antioxidants back to their reduced form. MDHAR and GR use NADPH, whereas DHAR uses GSH as a reducing equivalent (Foyer and Noctor 2009).

The significance of the key antioxidant enzymes in plant defence responses has been demonstrated by thousands of publications, but only a few studies have investigated their role under night or artificial dark conditions. When plants are in the darkness, it is particularly important to understand the gene expression profile as well as any changes in the activity and subcellular localization of antioxidant enzymes in the case of many developmental processes (germination, growth, and senescence) and under different environmental stresses (cold, chilling, heat, and biotic stress). Moreover, the regulation of antioxidant enzymes by phytohormones can also be different in the dark compared to the light conditions. 


\section{Circadian and diurnal regulation of the key antioxidant enzymes}

Changes in antioxidant enzyme activities are dependent not only on plant tissues and organs, on plant developmental stages, or on the intensity of different stressors, but also on the presence or absence of light, which has received less attention. To understand the role of these enzymes under darkness, the circadian and/or diurnal regulation should be firstly recognized. Earlier it was shown that plants with Crassulacean acid metabolism (CAM) have a strong diurnal rhythm in the activity of some antioxidant enzymes compared to C3 plants (Ślesak et al. 2002). Mesembryanthemum crystallinum and Sedum species are able to switch their mode of photosynthesis from $\mathrm{C} 3$ to CAM depending on the availability of water in the dry season or other unfavourable environmental conditions such as salinity (Libik et al. 2005). The mitochondrial MnSOD shows the most pronounced fluctuation in $M$. crystallinum; its activity is greatly reduced during the night and strongly increases in the afternoon following the activation of the mitochondrial electron transport chain. The FeSOD shows similar changes during the day. However, the transcriptions of $C u / Z n S O D$ and FeSOD increase in the dark compared to the light period in these plants (Ślesak et al. 2002). It has been also found by other authors that the total SOD activity is higher in the evening (at dusk) than in the morning (at dawn) in M. crystallinum (Libik et al. 2005). In parallel with this, a strong increase in the activity of MnSOD and $\mathrm{Cu} / \mathrm{ZnSOD}$ after salt stress during the evening is observed in these plants, suggesting that salinity can induce oxidative stress in mitochondria and in the cytosol at this time (Libik et al. 2005). Sedum album and Sedum stoloniferum can also switch between C3 carbon fixation and CAM depending on the water availability. As C3 plants, they do not exhibit diurnal rhythms for SOD activity under control conditions, but the activity of SOD is higher during the evening hours under drought stress (Habibi and Hajiboland 2012). In a classic C3 species Nicotiana sylvestris, the transcription of FeSOD is slightly decreased, while the expression of MnSOD remains constant during the night period (Dutilleul et al. 2003). However, total SOD activity in Solanum lycopersicum leaves is the highest at the end of day and decreases during the night (Kerdnaimongkol et al. 1997). In addition, relative transcriptions of FeSOD and $M n S O D$ also decrease in the early hours of the dark period in tomato leaves (Poór et al. 2017). These data suggest that total SOD activity is determined by the various SOD isoenzymes which are regulated differently during the day. Moreover, no correlation can be observed between SOD isoenzyme activities and the respective gene transcription at all times. SOD activity is higher in stressed Mesembryanthemum and Sedum plants in the dark phase, but SOD activity and especially the transcription of FeSOD decrease in the tomato and tobacco plants in the night.

It is well established that CATs are subjected to photoinactivation under moderate irradiance, thus CATs also seem to be light-sensitive (Mhamdi et al. 2012). In addition, the transcriptional regulation of CAT isoenzymes also shows circadian and/or diurnal rhythm (Mhamdi et al. 2010). Based on several investigations, the peak of CAT3 mRNA abundance occurs in the evening, which is out of phase with the expression of CAT2 showing circadian-regulated expression connected to the early morning in Arabidopsis (Zhong and McClung 1996, Zhong et al. 1997, Michael and McClung 2002). Oryza sativa also contains three CAT genes (CatA, CatB, CatC). The expression of CatA in the leaf sheath is modulated by circadian rhythm with the maximal transcription at the end of the light period. On the other hand, diurnal oscillation is detected in the case of the CatC expression pattern in the leaf blade when plants are grown in the dark (Iwamoto et al. 2000, Joo et al. 2014). The maize CAT3 is also regulated by circadian rhythm showing maximal transcript accumulation late in the light period and low in the late dark and early light periods (Boldt and Scandalios 1995). Morning-specific circadian regulation of CAT1 is observed in Capsicum annuum, where the expression of $C A T 1$ reaches its maximum late in the dark period or early in the light period (Kwon and An 2001). Diurnal regulation of CAT1 and CAT2 mRNA abundance has been observed in Triticum aestivum, where the abundance of the CAT1 transcripts is regulated by circadian rhythm that also persists in continuous darkness, whereas CAT2 is modulated by light (Luna et al. 2005). In Prunus persica leaves, CAT1 is responsive to irradiance and to seasonal changes, whereas $C A T 2$ displays high expression in shoots and is responsive only to seasonal changes (Bagnoli et al. 2004). Transcript abundance of CAT1 in cultivated tobacco decreases during the light period and it is very low at the end of the day, but it increases again during the dark period. In contrast to $C A T 1$, transcriptions of $C A T 2$ and $C A T 3$ show opposite diurnal changes. Expressions of $C A T 2$ and $C A T 3$ increase by 6 - to 7 -fold during the light period and markedly decrease during darkness (Dutilleul et al. 2003). In tomato, the transcription of CAT1 is high late in the light period and early in the dark period, whereas CAT2 expression is high late in the dark phase and early in the light phase (Kabir and Wang 2011, Poór et al. 2017). In CAM plants like in C3 plants, CAT activity also shows daily fluctuations, with the maximum at the end of the light period (Niewiadomska and Miszalski 2008). A similar tendency in CAT activity has been observed in S. album and S. stoloniferum, especially under drought stress (Habibi and Hajiboland 2012). It can be concluded that the transcriptional regulation and the activity of CAT isoenzymes depend on the phase of the circadian or diurnal cycle and on plant species. Several $C A T$ genes show maximal transcript accumulation late in the light and low late in the dark and early in the light period. Similar to the regulation of transcription, Kerdnaimongkol et al. (1997) found that the total CAT activity in tomato leaves is the highest late in the light 
period and it is lower during the night.

The different APX isoenzymes show differences not only in subcellular but also in light-dependent activity. It has been found in cultivated tobacco that two APX isoforms located in the cytosol (cAPX1 and cAPX2) are activated differently in the presence or absence of light. Namely, the transcription of $c A P X 1$ decreases after $16 \mathrm{~h}$ in the dark but it increases in the light (Pignocchi et al. 2003). Earlier it was observed that the $c A P X 1$ mRNA abundance increases during the day and decreases during the night in Arabidopsis (Kubo et al. 1995) and in tomato leaves (Poór et al. 2017). Similarly, the activity of APX as well as the relative transcription of chloroplastic and cytosolic APX is decreased during the night in Nicotiana sylvestris leaves (Dutilleul et al. 2003). Moreover, it has been also confirmed that the expression of both chloroplastic $S A P X$ and $t A P X$ show similar tendencies in tobacco leaves in a dark environment (Pignocchi et al. 2003). APX activity can be different in various plant organs and also shows diurnal changes in the exocarp of

\section{Seedling growth and development in the dark}

The activity of the antioxidant enzymes is also dependent on the developmental stages of plants. Darkness plays a pivotal role during skotomorphogenesis, when seedlings are growing and developing in the soil to seek the light (Josse and Halliday 2008). Elongation of the hypocotyl can be observed at the expense of cotyledon and root development during this process. Skotomorphogenesis is mediated by phytohormones acting on $\mathrm{H}_{2} \mathrm{O}_{2}$ homeostasis (Wojtyla et al. 2016) and by other signalling components like transcription factors (e.g., phytochrome interacting factor, PIF), the key modulators in the dark (Leivar and Quail 2011). Kaur et al. (2009) found that SOD activity is lower but APX activity is higher in dark-germinated 3-d-old Cicer arietinum seedlings compared to the irradiated ones. However, CAT activity is higher in the cotyledons of etiolated seedlings of chickpea compared to the shoot, but irradiance results in up-regulation of CAT activity in all organs of this plant. In addition, biomass of shoots and roots decreases under the light (Kaur et al. 2009). Similarly, SOD activity is lower but CAT activity is higher in dark-germinated Brassica napus than in that germinated in the light for $5 \mathrm{~d}$. The APX activities in these plants do not differ between the two environments. However, the application of the germination inhibitor methyl jasmonate (MeJA) does not change SOD and CAT activities, but it enhances APX activity in the dark (Comparot et al. 2002). Furthermore, SOD activity does not change but CAT activity increases during Helianthus annuus seedling growth at $15{ }^{\circ} \mathrm{C}$ in the dark, but both enzyme activities increase after promoted ageing $(5 \mathrm{~d}$ at fruits. Low APX activity is observed in the early morning and high activity is in the middle of the day in apple fruit peels (Cheng and Ma 2004). These results have confirmed that APX plays a role mostly in the light phase of the day. In contrast, Ślesak et al. (2002) observed higher $A P X$ transcription in the dark than in light phases in $M$. crystallinum plants. This result also suggests that day/night changes of $A P X$ transcription in CAM plants exhibit an inverse pattern relative to that observed in $\mathrm{C} 3$ plants, where APX activity decreases in the dark.

The dynamics of $\mathrm{H}_{2} \mathrm{O}_{2}$ content is dependent on many diverse external and internal factors. Moreover, there is a close relationship between the content of $\mathrm{H}_{2} \mathrm{O}_{2}$ and the activity of antioxidant enzymes (Luna et al. 2005). Since photochemical formation of $\mathrm{H}_{2} \mathrm{O}_{2}$ can be more significant in the light phase of the day, thus light-dependent regulation of the key antioxidant enzymes serves to limit excessive $\mathrm{H}_{2} \mathrm{O}_{2}$ accumulation while allowing essential signalling functions to occur.

$45{ }^{\circ} \mathrm{C}$ and $100 \%$ relative humidity) (Bailly et al. 2002). Based on these results, SOD activity is reduced but CAT and APX activities increase or do not change in the dark during seed germination and early seedling growth and development (Fig. 1.). This process is dependent on the absence of light and mediated by several phytohormones and $\mathrm{H}_{2} \mathrm{O}_{2}$ (Wojtyla et al. 2016).

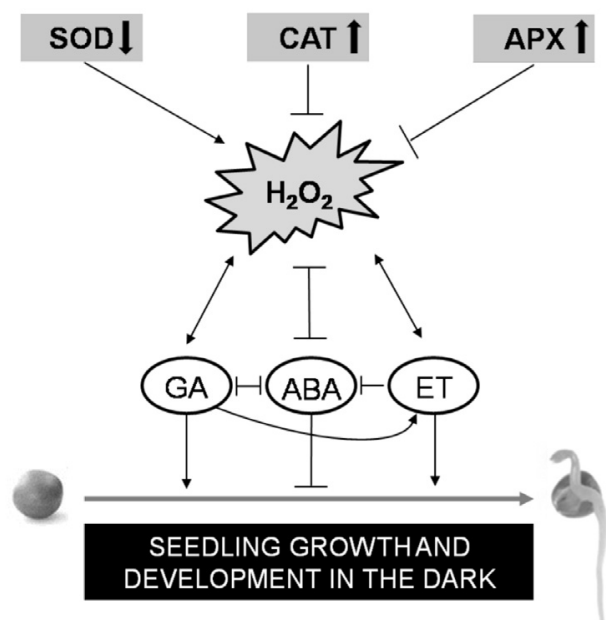

Fig. 1. Crosstalk between $\mathrm{H}_{2} \mathrm{O}_{2}$ homeostasis regulated by the key antioxidant enzymes and interactions between phytohormones for seedling growth and development in the dark. ABA - abscisic acid; APX - ascorbate peroxidase; CAT - catalase; ET - ethylene; GA - gibberellic acid; SOD superoxide dismutase. 


\section{Dark-induced senescence}

Senescence is an important physiological and molecular process, which can be induced by darkness. Dark-induced senescence is well characterized by the loss of chlorophyll and proteins, by enhanced ROS production, or by increased lipid peroxidation and membrane permeability. Changes in phytohormones, such as the induction of ethylene (ET) emission and the drop in the content of cytokinins (CKs), are also important regulators of this process, since these two hormones function as the main controllers of senescence (Luschin-Ebengreuth and Zechmann 2016, Cavaiuolo et al. 2017). From this aspect, the role of antioxidant enzymes in controlling ROS accumulation is particularly important in darkinduced senescence. Similar problems can emerge in the case of commercial shipping or storage of flowers, fruits, and vegetables (Ghorbanpour and Hatami 2014, Cavaiuolo et al. 2017).

It has been also shown that there are differences in the role of antioxidant enzyme activities between natural and dark-induced senescence. It has been found that SOD activity decreases but APX activity increases in Cucumis sativus cotyledons upon natural senescence (Kanazawa et al. 2000). Similarly, the protein content of mitochondrial MnSOD also decreases, suggesting the progression of mitochondrial senescence (Kanazawa et al. 2000). On the other hand, elevated induction of peroxisomal SOD has been reported during dark-induced senescence (Kanazawa et al. 2000). Interestingly, the CAT protein content transiently decreases in the early stages of natural senescence, but increases in the later stages, contrary to the CAT activity, which continuously decreases in cucumber cotyledons (Kanazawa et al. 2000). Decreased mitochondrial MnSOD activity and increased peroxisomal MnSOD activity are also revealed in dark-treated Pisum sativum leaves from the $3^{\text {rd }}$ day (Del Rio et al. 2002). These results show that SOD isoforms of different locations may have diverse functions during dark-induced senescence. In addition, APX activity also shows transient changes, because it increases after $4 \mathrm{~d}$ but decreases significantly after $6 \mathrm{~d}$ during dark-induced senescence in wheat leaves (Zavaleta-Mancera et al. 2007). Other authors found the same tendencies: APX activity increases in the first 7 to $10 \mathrm{~d}$ in the dark in Arabidopsis, but CAT activity decreases after $2 \mathrm{~d}$ of dark incubation (LuschinEbengreuth and Zechmann 2016). Similarly, CAT activity decreases upon darkness in chickpea (Sheokand and Swaraj 1996), but elevated APX activity is revealed in tomato leaves after $2 \mathrm{~d}$ in the dark (Kuzniak 2004). Furthermore, darkness induces a moderate increase in the total SOD but decreases CAT and APX activities in nodules of Lupinus albus after $7 \mathrm{~d}$ (Hernández-Jiménez et al. 2002). Dark-induced senescence elevates ${ }^{\circ} \mathrm{O}_{2}{ }^{-}$and $\mathrm{H}_{2} \mathrm{O}_{2}$ content in detached leaves of perennial ryegrass after $8 \mathrm{~d}$, and simultaneously the activity of CAT decreases, however, the transcription of CAT and APX activity rises (Zhang et al. 2016). The transcription of
$C A T$ also increases in peach leaves after $36 \mathrm{~h}$ in the dark (Giannino et al. 2004). It can be concluded that darkinduced senescence basically decreases CAT activity, but in some cases it has been shown that the expression of several $C A T$ genes increase. Based on these results, CAT activity decreases in the course of both natural and darkinduced senescence.

The effect of prolonged darkness on antioxidant enzymes of four cultivars of Pelargonium zonale was investigated after 4-d-long dark storage (Ghorbanpour and Hatami 2014). Interestingly, SOD activity did not change significantly between the geranium cultivars kept under normal condition. However, if they were kept in the dark, it was shown that after treatment with $60 \mathrm{mg} \mathrm{dm}^{-3}$ silver nanoparticles and/or $75 \mu \mathrm{M}$ thidiazuron (1-phenyl-3- (1,2,3-thiadiazol-5-yl) urea, TDZ), SOD, CAT, and APX activities were increased. Silver nanoparticles were able to inhibit ET signalling and decrease the degradation of chlorophyll content under the darkness (Ghorbanpour and Hatami 2014). In contrast, expression of CAT1 is enhanced temporarily by prolonged dark period $(6 \mathrm{~d})$ and by treatment with $1 \mathrm{mM}$ ET donor ethephon (2-chloroethylphosphonic acid) in Ipomoea batatas (Chen et al. 2012).

Other phytohormones such as 6-benzylaminopurine (BAP), a synthetic $\mathrm{CK}$, can also regulate antioxidant enzyme activities under dark-induced senescence. The $10 \mu \mathrm{M}$ BAP induces SOD, CAT, and APX activities and alleviates senescence in detached wheat leaves during dark incubation for $48 \mathrm{~h}$ (Huang et al. 2011). A similar tendency was observed in wheat leaves, where the decrease of CAT activity is slightly restored by the application of $100 \mu \mathrm{M}$ BAP after $4 \mathrm{~d}$ in darkness (Zavaleta-Mancera et al. 2007). The APX activity is also enhanced by BAP, which alleviates dark-induced senescence in wheat leaves under darkness (ZavaletaMancera et al. 2007).

In contrast, treatment with $5 \mathrm{mg} \mathrm{dm}^{-3}$ paclobutrazol (1-(4-chlorophenyl)-4,4-dimethyl-2-(1,2,4-triazol-1-yl) pentan-3-ol, PBZ), which can suppress the synthesis of gibberellic acids (GA) does not change SOD but increases CAT activity during dark-induced senescence of Vigna radiata, where dark for $5 \mathrm{~d}$ decreases CAT activity in the untreated plants (Bora et al. 2007). These results suggest that GA has only a little effect on SOD activity and did not affect the CAT activity during darkinduced senescence in these plants (Bora et al. 2007).

Moreover, treatment with $1 \mu \mathrm{M}$ naphthalene acetic acid (NAA) inhibits the decrease of CAT activity in detached leaves of Tropaeolum majus from the second day of the dark-induced senescence (Karatas et al. 2010).

Protective effects were also observed by the use of sodium nitroprusside (SNP), a nitric oxide donor, in detached leaves of Malus hupehensis after $3 \mathrm{~d}$ in the dark, where treatment with $100 \mu \mathrm{M}$ SNP alleviates lipid peroxidation and increases SOD, CAT, and APX 
activities (Gao et al. 2010).

Moreover, application of $100 \mu \mathrm{M}$ melatonin also enhances the activity of SOD and CAT in detached leaves of Lolium perenne after $8 \mathrm{~d}$ and delays leaf senescence (Zhang et al. 2016). Interestingly, $50 \mathrm{mg} \mathrm{dm}^{-3}$ selenium treatment similarly improves the adverse effects of darkinduced senescence by increasing CAT activity in mung bean (Moussa and Ahmed 2010).

It can be concluded that applications of BAP and SNP moderate the decrease in SOD, CAT, and APX activities under darkness, which can delay dark-induced senescence in several plant species. Nevertheless, application of ET receptor blocker (silver nanoparticles), GA synthesis inhibitor PBZ, synthetic auxin NAA, melatonin, or selenium can delay dark-induced senescence by increasing the activity of CAT (Fig. 2). These results confirm the fact of the hormonal regulation of antioxidant enzyme activities under darkness.

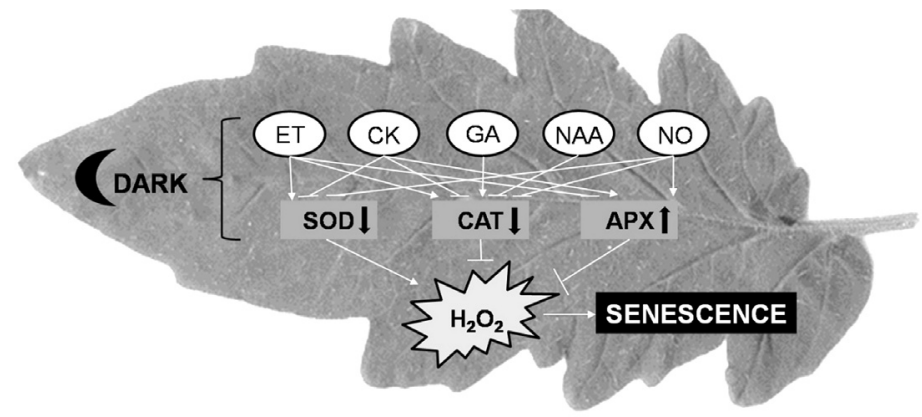

Fig. 2. Effects of phytohormones and growth regulators on the key antioxidant enzymes and $\mathrm{H}_{2} \mathrm{O}_{2}$ homeostasis during dark-induced senescence. APX - ascorbate peroxidase; ET - ethylene; CAT - catalase; CK - cytokinin; ET - ethylene; GA - gibberellic acid; NAA naphthaleneacetic acid; NO - nitric oxide; SOD - superoxide dismutase.

\section{Plant stress responses in the dark}

Dark can also modulate the responses to several stresses (e.g., cold, chilling, and heat). Chilling and cold stress can induce rapid production of ROS, thus plants have to enhance the activity of antioxidant enzymes to protect themselves especially against the destruction of cell membranes. Usually, plants are exposed to lower temperature during the night. However, the effect of chilling or cold stress in the dark is usually not as severe as the same temperature in the light (Novák et al. 2015). In Crocus sativus, SOD, CAT, and APX activities are significantly higher under low temperature $\left(5^{\circ} \mathrm{C}\right)$ in the dark compared to those in the light (Esfandiari et al. 2009). Moreover, activities of SOD, CAT, and APX increases significantly in the leaves of Cattleya and Cymbidium plants exposed to $4{ }^{\circ} \mathrm{C}$ for $24 \mathrm{~h}$ in the dark compared to non-chilled leaves kept at $28{ }^{\circ} \mathrm{C}$ ( $\mathrm{Li}$ and Matsui 2001). In addition, activities of SOD and APX are slightly increased by a $24-\mathrm{h}$ cold treatment $\left(10^{\circ} \mathrm{C}\right)$ in the dark in Medicago sativa (Bafeel and Ibrahim 2008). The activity of CAT is variable depending on the duration of chilling, developmental stage, and plant organ (Wojciechowska et al. 2013). Although CAT activity decreases after chilling stress in the first week at $2{ }^{\circ} \mathrm{C}$ under darkness, it does not change after the second week compared to the control in Brassica oleracea seedlings (Wojciechowska et al. 2013). Interestingly, the important stress hormone abscisic acid (ABA) also promoted SOD, CAT, and APX activities after $7 \mathrm{~d}$ in moderated coldstressed $\left(15^{\circ} \mathrm{C}\right)$ shoots of maize seedlings under dark conditions (Khorshidi and Nojavan 2006). In addition, 2 weeks at $4{ }^{\circ} \mathrm{C}$ do not induce changes in CAT activity in the leaves of Lilium plants, but shifting from 4 to $22{ }^{\circ} \mathrm{C}$ induce rapid loss of chlorophyll, proteolysis, increased lipid peroxidation, and decreased CAT activity, which can be prevented by $100 \mathrm{mg} \mathrm{dm}^{-3}$ GA treatment (Ranwala et al. 2000). To summarize, enhanced SOD, CAT and APX activities are a common phenomenon in coldstressed plants in the dark, but changes in antioxidant enzyme activities are dependent not only on the development of cold stress and plant species or organs but also on the presence of several phytohormones such as ABA or GA (Fig. 3.).

Other abiotic stresses such as high temperature and heat shock can also inhibit photosynthesis, limit sugar accumulation, or destruct cell membranes and cytoskeleton. Total SOD activity is slightly increased in tomato plants that have been subjected to both heat acclimation $\left(35^{\circ} \mathrm{C}\right)$ and heat shock $\left(45^{\circ} \mathrm{C}\right)$ for $3 \mathrm{~d}$ in the dark (Camejo et al. 2007). Activities of FeSOD isoenzymes are enhanced significantly in heat-stressed tomato plants under dark conditions. Moreover, MnSOD activity is also raised but the $\mathrm{Cu} / \mathrm{ZnSOD}$ activity is decreased in the dark at $35^{\circ} \mathrm{C}$ (Camejo et al. 2007). In addition, it has been observed in cultivated tobacco that SOD activity remains unchanged until the temperature is elevated to $45^{\circ} \mathrm{C}$ for $4 \mathrm{~h}$, and subsequently shows an increasing tendency during heating to $50{ }^{\circ} \mathrm{C}$, but CAT activity decreases in parallel (Yang et al. 2007). Similarly, CAT activity decreases in the leaves of pepper exposed to 48 and $54{ }^{\circ} \mathrm{C}$ for $15 \mathrm{~min}$ in the dark (Anderson 2002). In addition, high night temperature is also a significant problem, which can cause reduction of 
yield in some important crops. Shah et al. (2011) observed an increase in CAT activity in rice leaves after high night temperature $\left(30^{\circ} \mathrm{C}\right)$, which can be moderated by application of $5 \mathrm{mM}$ ascorbic acid. Compared to the cold stress, heat stress has an opposite effect on APX activity. Significantly lower APX activity is observed in leaves of Fagus sylvatica collected in warm nights in comparison with those collected in cooler nights (Peltzer and Polle 2001). Similar tendencies are found in cultivated tobacco, where APX activity significantly decreases upon increasing the temperature (above $35^{\circ} \mathrm{C}$ ), and this effect is more pronounced in the light than in the dark (Yang et al. 2007). Besides, the activities of $\mathrm{Cu} / \mathrm{ZnSOD}$, FeSOD, and CAT are significantly decreased by heat shock $\left(45^{\circ} \mathrm{C}\right.$ for $90 \mathrm{~min}$ in dark) in leaf discs of mung bean, whereas this effect is prevented by presoaking with $150 \mu \mathrm{M}$ SNP (Yang et al. 2006). High temperature and heat shock also elevate SOD but decrease CAT and APX activities in the dark. However, these changes can be modulated by the duration and severity of the stress, and they are also dependent on the plant species (Fig. 3.).

After having acknowledged the significance of various abiotic stress conditions, it has to be noted that the role of antioxidant enzymes during biotic stress is also crucial in the defence responses of plants. The time (e.g. at night) and the presence or absence of light can also be crucial in case of herbicide application on crops. Paraquat (1,1'-dimethyl-4,4'-bipyridinium dichloride) is an important non-selective herbicide, which can interact with photosystem $I$ in the light via induction of superoxide free radical formation, thus causing lethal oxidative stress in plants. However, it was found that $100 \mu \mathrm{M}$ Paraquat does not change SOD activity in the first $12 \mathrm{~h}$ of the dark treatment in Salvia officinalis (Radyukina et al. 2008). In contrast, some chemicals, such as the auxin-type herbicide Quinclorac (3,7-dichloro-8-quinoline-carboxylic acid), are selective for different plant species. Since Quinclorac induces the activity of 1-aminocyclo-propane-1-carboxylic acid (ACC) synthase, one of the key enzymes of ET biosynthesis, and thereby increases the accumulation of ethylene, cyanide, and ROS in the tissues of susceptible grasses, it could be expected that its activity depends on plant irradiance. The herbicide-induced oxidative damage in susceptible grass species and the differences in antioxidative ability can be crucial factors in the mode of their action. However, $1 \mu \mathrm{M}$ Quinclorac does not affect the SOD, CAT, and APX activities during the first $24 \mathrm{~h}$ of the application in rice and Echinochloa oryzicola plants under both light and dark conditions (Sunohara and Matsumoto 2004). These investigations can be crucial for optimizing the use of plant herbicides in the agriculture.

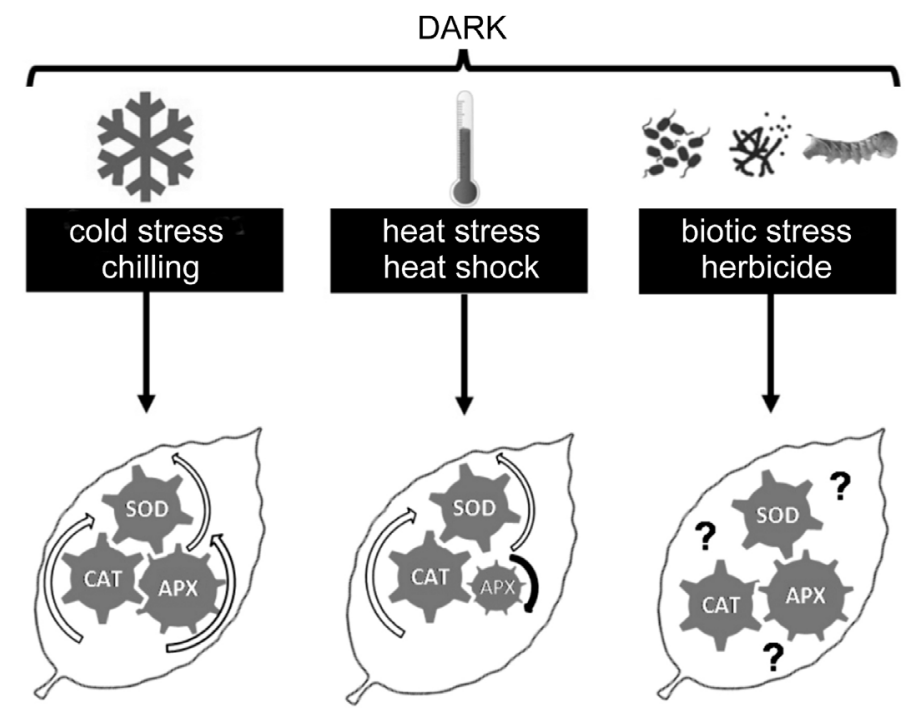

Fig. 3. Stress-induced changes in the key antioxidant enzymes in the leaves of various plant species under darkness. APX - ascorbate peroxidase, CAT - catalase, SOD - superoxide dismutase. White arrow means an increase, black arrow means a decrease in antioxidant activity, and question mark means unexplored role of antioxidant enzyme activity.

\section{Conclusions and perspectives}

Despite the fact that the role of antioxidant enzymes has been intensively studied in the past twenty years, there are still many gaps in comprehending the alteration of antioxidant enzyme activities in the dark. In this review, we highlighted the current state of the physiological and molecular aspects of dark-modulated key antioxidant enzymes in different plant species and discussed the role of SOD, CAT, and APX in various developmental processes (seedling growth and development or senescence) and under different environmental stresses 
(cold, chilling, heat, and biotic stress) in the absence of light. Moreover, the regulation of these antioxidant enzymes by phytohormones and the changes in the activity of isoenzymes on subcellular level in the dark were also summarized.

The SOD, CAT, and APX activities show diurnal regulation. Moreover, the specific subcellular activity can also be crucial under darkness. Several CAT genes show maximal transcript accumulation late in the light period and low late in the dark and early in the light period. In addition, the relative transcriptions of chloroplastic and cytosolic $A P X$ decrease during the night, whereas these transcript patterns are at their maximum in the light. In contrast, CAM plants show an inverse pattern of these activities. Darkness can also modulate the activities of antioxidant enzymes during seedling growth and development in the dark or during dark-induced senescence. The SOD activity is lower, but CAT activity is higher during the early seedling growth and development. The APX activity is enhanced or does not change in dark-germinated seedlings. In the case of senescence, mitochondrial MnSOD decreases but peroxisomal MnSOD increases in the dark. CAT activity also declines, but APX activity shows a transient increase in dark-induced senescent leaves. Application of cytokinin BAP or NO donor SNP can delay dark-induced senescence by increasing all investigated antioxidant enzymes in the dark. It can be concluded that the activity of antioxidant enzymes depends on the duration of cold stress as well as the plant species and organs. The SOD and APX activities are significantly increased upon low temperature under darkness. High night temperature enhances SOD and CAT, but decreases APX activity. Interestingly, Quinclorac, an auxin-type herbicide, does not change any of the investigated antioxidant enzyme activities during the first $24 \mathrm{~h}$ of darkness and Paraquat also does not affect SOD activity in the first $12 \mathrm{~h}$ in the

\section{References}

Alscher, R.G., Erturk, N., Heath, L.S.: Role of superoxide dismutases (SODs) in controlling oxidative stress in plants. - J. exp. Bot. 53: 1331-1341, 2002.

Anderson, J.A.: Catalase activity, hydrogen peroxide content and thermotolerance of pepper leaves. - Sci. Hort. 95: 277284, 2002.

Asada, K.: The role of ascorbate peroxidase and monodehydroascorbate reductase in $\mathrm{H}_{2} \mathrm{O}_{2}$ scavenging in plants. - Cold Spring Harbor Monograph Archive 34: 715-735, 1997.

Bafeel, S.O., Ibrahim, M.M.: Antioxidants and accumulation of $\alpha$-tocopherol induce chilling tolerance in Medicago sativa. Int. J. Agr. Biol. 10: 593-598, 2008.

Bagnoli, F., Danti, S., Magherini, V., Cozza, R., Innocenti, A.M., Racchi, M.L.: Molecular cloning, characterisation and expression of two catalase genes from peach. - Funct. Plant Biol. 31: 349-357, 2004.

Bailly, C., Bogatek-Leszczynska, R., Côme, D., Corbineau, F.: Changes in activities of antioxidant enzymes and lipoxygenase during growth of sunflower seedlings from seeds of different vigour. - Seed Sci. Res. 12: 47-55, 2002. dark.

Unfortunately, most of the authors determined the activity of antioxidant enzymes only at one time-point after stress treatments in the dark, however, the enzyme activities may vary from some hours to a few days or even weeks. Moreover, antioxidant enzymes can be activated not only in a time- but also in tissue-specific manner under dark conditions. In addition, these antioxidant enzymes may reside at different subcellular locations, where they may be differentially regulated in the dark. Unfortunately, many authors investigated only the total activity of antioxidant enzymes and did not analyze the different isoenzymes. In this regard, further research of the organ and temporal regulation of antioxidant enzyme activity and respective gene expression is necessary for understanding their regulation in the dark. Moreover, cross-talk among hormones under dark conditions is also worth of further investigation. It can be concluded that the timing of the application of phytohormones (e.g., BAP, NAA, ABA), modulators (e.g., NO donor SNP, ET inhibitor silver nanoparticles), and herbicides can determine their effects on the plants, because of the different circadian regulation of antioxidant enzymes. In the future, the accurate description of the specificity of these antioxidant enzymes in the signal transduction of plant hormones in the dark would also provide new insights into converging and diverging signalling pathways under diverse environmental conditions.

Understanding the mechanism that can regulate antioxidant enzymes at the molecular, cellular, tissue, or whole plant levels is an important problem in current plant biology as well as in agriculture. Based on this knowledge, the modification of these antioxidant enzymes may be used to increase the yield and stress tolerance under changing environment.
Ballaré, C.L.: Light regulation of plant defense. - Annu. Rev. Plant Biol. 65: 335-363, 2014.

Boldt, R., Scandalios, J.G.: Circadian regulation of the Cat3 catalase gene in maize (Zea mays L.): entrainment of the circadian rhythm of Cat3 by different light treatments. Plant J. 7: 989-999, 1995.

Bora, K.K., Ganesh, R., Mathur, S.R.: Paclobutrazol delayed dark-induced senescence of mung bean leaves. - Biológia 62: 185-188, 2007.

Camejo, D., Martí, M.D.C., Nicolás, E., Alarcón, J.J., Jiménez, A., Sevilla, F.: Response of superoxide dismutase isoenzymes in tomato plants (Lycopersicon esculentum) during thermo-acclimation of the photosynthetic apparatus. - Physiol. Plant. 131: 367-377, 2007.

Cavaiuolo, M., Cocetta, G., Spadafora, N. D., Müller, C. T., Rogers, H. J., Ferrante, A.: Gene expression analysis of rocket salad under pre-harvest and postharvest stresses: a transcriptomic resource for Diplotaxis tenuifolia. - PloS ONE 12: e0178119, 2017.

Caverzan, A., Passaia, G., Rosa, S.B., Ribeiro, C.W., 
Lazzarotto, F., Margis-Pinheiro, M.: Plant responses to stresses: role of ascorbate peroxidase in the antioxidant protection. - Genet. mol. Biol. 35: 1011-1019, 2012.

Chen, H.J., Wu, S.D., Huang, G.J., Shen, C.Y., Afiyanti, M., Li, W.J., Lin, Y.H.: Expression of a cloned sweet potato catalase SPCAT1 alleviates ethephon-mediated leaf senescence and $\mathrm{H}_{2} \mathrm{O}_{2}$ elevation. - J. Plant Physiol. 169: 8697, 2012.

Chen, M., Chory, J., Fankhauser, C.: Light signal transduction in higher plants. - Annu. Rev. Genet. 38: 87-117, 2004.

Cheng, L., Ma, F.: Diurnal operation of the xanthophyll cycle and the antioxidant system in apple peel. - J. amer. Soc. hort. Sci. 129: 313-320, 2004

Comparot, S.M., Graham, C.M., Reid, D.M.: Methyl jasmonate elicits a differential antioxidant response in light-and darkgrown canola (Brassica napus) roots and shoots. - Plant Growth Regul. 38: 21-30, 2002.

Del Rio, L.A., Sandalio, L.M., Altomare, D.A., Zilinskas, B.A.: Mitochondrial and peroxisomal manganese superoxide dismutase: differential expression during leaf senescence. J. exp. Bot. 54: 923-933, 2003.

Dutilleul, C., Garmier, M., Noctor, G., Mathieu, C., Chétrit, P., Foyer, C.H., De Paepe, R.: Leaf mitochondria modulate whole cell redox homeostasis, set antioxidant capacity, and determine stress resistance through altered signaling and diurnal regulation. - Plant Cell 15: 1212-1226, 2003.

Esfandiari, E., Alavi-Kia, S.S., Bahmani, A., Aazami, M.A.: The effect of light on ROS-scavenging systems and lipid peroxidation under cold conditions in saffron (Crocus sativus L.). - Afr. J. agr. Res. 4: 378-382, 2009.

Foyer, C. H., Noctor, G.: Oxygen processing in photosynthesis: regulation and signaling. - New Phytol. 146: 359-388, 2000.

Foyer, C.H., Noctor, G.: Redox regulation in photosynthetic organisms: signaling, acclimation, and practical implications. - Antioxid. Redox Signal. 11: 861-905, 2009.

Gao, H.J., Zhao, F., Yang, J.X., Yang, H.Q.: Nitric oxide alleviates lipid peroxidation induced by osmotic stress during senescence of detached leaves of Malus hupehensis Rehd. - J. hort. Sci. Biotechnol. 85: 367-373, 2010.

Ghorbanpour, M., Hatami, M.: Spray treatment with silver nanoparticles plus thidiazuron increases anti-oxidant enzyme activities and reduces petal and leaf abscission in four cultivars of geranium (Pelargonium zonale) during storage in the dark. - J. hort. Sci. Biotechnol. 89: 712-718, 2014.

Giannino, D., Condello, E., Bruno, L., Testone, G., Tartarini, A., Cozza, R., Mariotti, D.: The gene geranylgeranyl reductase of peach (Prunus persica [L.] Batsch) is regulated during leaf development and responds differentially to distinct stress factors. - J. exp. Bot. 55: 2063-2073, 2004.

Habibi, G., Hajiboland, R.: Comparison of photosynthesis and antioxidative protection in Sedum album and Sedum stoloniferum (Crassulaceae) under water stress. Photosynthetica 50: 508-518, 2012.

Hernández-Jiménez, M.J., Lucas, M.M., de Felipe, M.R.: Antioxidant defence and damage in senescing lupin nodules. - Plant Physiol. Biochem. 40: 645-657, 2002.

Huang, J., Han, B., Xu, S., Zhou, M., Shen, W.: Heme oxygenase- 1 is involved in the cytokinin-induced alleviation of senescence in detached wheat leaves during dark incubation. - J. Plant Physiol. 168: 768-775, 2011.

Iwamoto, M., Higo, H., Higo, K.: Differential diurnal expression of rice catalase genes: the 5 -flanking region of CatA is not sufficient for circadian control. - Plant Sci. 151: 39-46, 2000.
Joo, J., Lee, Y.H., Song, S.I.: Rice CatA, CatB, and CatC are involved in environmental stress response, root growth, and photorespiration, respectively. - J. Plant Biol. 57: 375-382, 2014.

Josse, E.M., Halliday, K.J.: Skotomorphogenesis: the dark side of light signalling. - Curr. Biol. 18: 1144-1146, 2008.

Kabir, M.H., Wang, M.H.: Functional studies on two catalase genes from tomato (Solanum lycopersicum L.). - J. hort. Sci. Biotechnol. 86: 84-90, 2011.

Kanazawa, S., Sano, S., Koshiba, T., Ushimaru, T.: Changes in antioxidative enzymes in cucumber cotyledons during natural senescence: comparison with those during darkinduced senescence. - Physiol. Plant. 109: 211-216, 2000.

Kangasjärvi, S., Neukermans, J., Li, S., Aro, E.M., Noctor, G.: Photosynthesis, photorespiration, and light signalling in defence responses. - J. exp. Bot. 63: 1619-1636, 2012.

Karatas, I., Oztürk, L., Ersahin, Y., Okatan, Y.: Effects of auxin on photosynthetic pigments and some enzyme activities during dark-induced senescence of Tropaeolum leaves. Pak. J. Bot. 42: 1881-1888, 2010.

Karpinski, S., Gabtys, H., Mateo, A., Karpinska, B., Mullineaux, P.M.: Light perception in plant disease defence signalling. - Curr. Opin. Plant Biol. 6: 390-396, 2003.

Kaur, H., Gupta, A.K., Kaur, N., Sandhu, J.S.: Differential response of the antioxidant system in wild and cultivated genotypes of chickpea. - Plant Growth Regul. 57: 109-114, 2009.

Kazan, K., Manners, J.M.: The interplay between light and jasmonate signalling during defence and development. - J. exp. Bot. 62: 4087-4100, 2011.

Kerdnaimongkol, K., Bhatia, A., Joly, R.J., Woodson, W.R.: Oxidative stress and diurnal variation in chilling sensitivity of tomato seedlings. - J. amer. Soc. hort. Sci. 122: 485-490, 1997.

Khorshidi, M., Nojavan, A.M.: The effects of abscisic acid and $\mathrm{CaCl}_{2}$ on the activities of antioxidant enzymes under cold stress in maize seedlings in the dark. - Pak. J. biol. Sci. 9: 54-59, 2006.

Kocsy, G., Tari, I., Vanková, R., Zechmann, B., Gulyás, Z., Poór, P., Galiba, G.: Redox control of plant growth and development. - Plant Sci. 211: 77-91, 2013.

Kubo, A., Saji, H., Tanaka, K., Kondo, N.: Expression of Arabidopsis cytosolic ascorbate peroxidase gene in response to ozone or sulfur dioxide. - Plant mol. Biol. 29: 479-489, 1995.

Kuzniak, E.: Ascorbate and ascorbate-dependent enzymes in detached tomato leaves under conditions modulating the ascorbate pool. - Acta Physiol. Plant. 26: 327-333, 2004.

Kwon, S.I., An, C.S.: Molecular cloning, characterization and expression analysis of a catalase cDNA from hot pepper (Capsicum annuum L.). - Plant Sci. 160: 961-969, 2001.

Leivar, P., Quail, P.H.: PIFs: pivotal components in a cellular signaling hub. - Trends Plant Sci. 16: 19-28, 2011.

Li, J., Matsui, S.: Effects of chilling on antioxidative enzymes in leaves of Cattleya and Cymbidium. - J. jap. Soc. hort. Sci. 70: 360-365, 2001.

Libik, M., Konieczny, R., Surówka, E., Miszalski, Z.: Superoxide dismutase activity in organs of Mesembryanthemum crystallinum L. at different stages of CAM development. - Acta biol. cracov. Ser. Bot. 47: 199-204, 2005.

Luna, C.M., Pastori, G.M., Driscoll, S., Groten, K., Bernard, S., Foyer, C.H.: Drought controls on $\mathrm{H}_{2} \mathrm{O}_{2}$ accumulation, catalase (CAT) activity and $C A T$ gene expression in wheat. - J. exp. Bot. 56: 417-423, 2005. 
Luschin-Ebengreuth, N., Zechmann, B.: Compartment-specific investigations of antioxidants and hydrogen peroxide in leaves of Arabidopsis thaliana during dark-induced senescence. - Acta Physiol. Plant. 38: 1-15, 2016.

Michael, T.P., McClung, C.R.: Phase-specific circadian clock regulatory elements in Arabidopsis. - Plant Physiol. 130: 627-638, 2002.

Mhamdi, A., Queval, G., Chaouch, S., Vanderauwera, S., Van Breusegem, F., Noctor, G.: Catalase function in plants: a focus on Arabidopsis mutants as stress-mimic models. - J. exp. Bot. 61: 4197-4220, 2010.

Mhamdi, A., Noctor, G., Baker, A.: Plant catalases: peroxisomal redox guardians. - Arch. Biochem. Biophys. 525: 181-194, 2012.

Moussa, H.R., Ahmed, A.E.F.M.: Protective role of selenium on development and physiological responses of Vicia faba. Int. J. veg. Sci. 16: 174-183, 2010.

Niewiadomska, E., Miszalski, Z.: Partial characterization and expression of leaf catalase in the CAM-inducible halophyte Mesembryanthemum crystallinum L. - Plant Physiol. Biochem. 46: 421-427, 2008.

Novák, A., Boldizsár, Á., Ádám, É., Kozma-Bognár, L., Majláth, I., Båga, M., Tóth, B., Chibbar, R., Galiba, G.: Light-quality and temperature-dependent $C B F 14$ gene expression modulates freezing tolerance in cereals. - J. exp. Bot. 67: 1285-1295, 2015.

Panchuk, I.I., Zentgraf, U., Volkov, R.A.: Expression of the Apx gene family during leaf senescence of Arabidopsis thaliana. - Planta 222: 926-932, 2005.

Peltzer, D., Polle, A.: Diurnal fluctuations of antioxidative systems in leaves of field-grown beech trees (Fagus sylvatica): Responses to light and temperature. - Physiol. Plant. 111: 158-164, 2001.

Pignocchi, C., Fletcher, J.M., Wilkinson, J.E., Barnes, J.D., Foyer, C.H.: The function of ascorbate oxidase in tobacco. Plant Physiol. 132: 1631-1641, 2003.

Poór, P., Takács, Z., Bela, K., Czékus, Z., Szalai, G., Tari, I.: Prolonged dark period modulates the oxidative burst and enzymatic antioxidant systems in the leaves of salicylic acid-treated tomato. - J. Plant Physiol. 213: 216-226, 2017.

Radyukina, N.L., Shashukova, A.V., Shevyakova, N.I., Kuznetsov, V.V.: Proline involvement in the common sage antioxidant system in the presence of $\mathrm{NaCl}$ and paraquat. Russ. J. Plant Physiol. 55: 649-656, 2008.

Ranwala, A.P., Miller, W.B.: Preventive mechanisms of gibberellin 4+7 and light on low-temperature-induced leaf senescence in Lilium cv. Stargazer. - Postharvest Biol. Technol. 19: 85-92, 2000.

Shah, F., Huang, J., Cui, K., Nie, L., Shah, T., Wu, W., Chen, C.: Physiological and biochemical changes in rice associated with high night temperature stress and their amelioration by exogenous application of ascorbic acid (vitamin C). - Aust. J. Crop. Sci. 5: 1810-1816, 2011.

Sheokand, S., Swaraj, K.: Natural and dark-induced nodule senescence in chickpea: nodule functioning and $\mathrm{H}_{2} \mathrm{O}_{2}$ scavenging enzymes. - Biol. Plant. 38: 545-554, 1996.
Ślesak, I., Miszalski, Z., Karpinska, B., Niewiadomska, E., Ratajczak, R., Karpinski, S.: Redox control of oxidative stress responses in the C3-CAM intermediate plant Mesembryanthemum crystallinum. - Plant Physiol. Biochem. 40: 669-677, 2002.

Sunohara, Y., Matsumoto, H.: Oxidative injury induced by the herbicide quinclorac on Echinochloa oryzicola Vasing. and the involvement of antioxidative ability in its highly selective action in grass species. - Plant Sci. 167: 597-606, 2004.

Van Aken, O., Van Breusegem, F.: Licensed to kill: mitochondria, chloroplasts, and cell death. - Trends Plant Sci. 20: 754-766, 2015.

Vranová, E., Inzé, D., Van Breusegem, F.: Signal transduction during oxidative stress. - J. exp. Bot. 53: 1227-1236, 2002.

Wada, K., Tada, T., Nakamura, Y., Ishikawa, T., Yabuta, Y., Yoshimura, K., Shigeoka, S., Nishimura, K.: Crystal structure of chloroplastic ascorbate peroxidase from tobacco plants and structural insights into its instability. - J. Biochem. 134: 239-244, 2003.

Wojciechowska, R., Hanus-Fajerska, E.J., Kolton, A., Kaminska, I., Grabowska, A., Kunicki, E.: The effect of seedling chilling on glutathione content, catalase and peroxidase activity in Brassica oleracea L. var. italica. Acta Soc. Bot. Polon. 82: 243-248, 2013.

Wojtyla, Ł., Lechowska, K., Kubala, S., Garnczarska, M.: Different modes of hydrogen peroxide action during seed germination. - Front. Plant Sci. 7: 66, 2016.

Yang, J.D., Yun, J.Y., Zhang, T.H., Zhao, H.L.: Presoaking with nitric oxide donor SNP alleviates heat shock damages in mung bean leaf discs. - Bot. Stud. 47: 129-136, 2006.

Yang, X., Wen, X., Gong, H., Lu, Q., Yang, Z., Tang, Y., Lu, C.: Genetic engineering of the biosynthesis of glycinebetaine enhances thermotolerance of photosystem II in tobacco plants. - Planta 225: 719-733, 2007.

Zavaleta-Mancera, H.A., López-Delgado, H., Loza-Tavera, H., Mora-Herrera, M., Trevilla-García, C., Vargas-Suárez, M., Ougham, H.: Cytokinin promotes catalase and ascorbate peroxidase activities and preserves the chloroplast integrity during dark-senescence. - J. Plant Physiol. 164: 1572-1582, 2007.

Zhang, J., Li, H., Xu, B., Li, J., Huang, B.: Exogenous melatonin suppresses dark-induced leaf senescence by activating the superoxide dismutase-catalase antioxidant pathway and down-regulating chlorophyll degradation in excised leaves of perennial ryegrass (Lolium perenne L.). Front. Plant Sci. 7: 1500, 2016.

Zhong, H.H., McClung, C.R.: The circadian clock gates expression of two Arabidopsis catalase genes to distinct and opposite circadian phases. - Mol. gen. Genet. 251: 196-203, 1996.

Zhong, H.H., Resnick, A.S., Straume, M., McClung, C.R.: Effects of synergistic signaling by phytochrome A and cryptochrome1 on circadian clock-regulated catalase expression. - Plant Cell 9: 947-955, 1997. 